\title{
Prevalence of Dry Eye and its Subtypes in an Elderly Population with Cataracts in Indonesia
}

This article was published in the following Dove Press journal:

Clinical Ophthalmology

\author{
Nina Asrini Noor (D) \\ Tri Rahayu (D) ${ }^{1,2}$ \\ Tjahjono D Gondhowiardjo ${ }^{1,2}$
}

'JEC Eye Hospitals and Clinics, Jakarta, Indonesia; ${ }^{2}$ Ophthalmology Department, Cipto Mangunkusumo Kirana Hospital, Jakarta, Indonesia
Correspondence: Nina Asrini Noor JEC Eye Hospital Kedoya, Jalan Terusan Arjuna Utara Nomor I, Jakarta Barat, DKI, Jakarta II 520, Indonesia

Tel +62 81395209670

Email nina.noor@jec.co.id
Purpose: To describe the prevalence of dry eye disease (DED) and its subtypes in elderly population with cataracts prior to surgery in Jakarta, Indonesia.

Patients and Methods: Forty-nine eyes of 49 patients (16 males, 33 females; mean age: 66.6 years) planned for phacoemulsification surgery were consecutively recruited from two tertiary eye hospitals in this hospital-based cross-sectional study. Symptoms were assessed using the Ocular Surface Disease Index (OSDI) questionnaire, and the patient was defined as symptomatic if their OSDI score was $\geq 13$. Objective examinations included the noninvasive tear film break up time (NIBUT) and Schirmer tests. The results were deemed abnormal if they were $<10$ seconds and $<10 \mathrm{~mm}$ for NIBUT and Schirmer, respectively.

Results: Participants were classified into four groups: DED (positive symptoms and short NIBUT), preclinical dry eye (positive symptoms without short NIBUT), predisposition to dry eye (short NIBUT without symptoms), and normal group. Median OSDI score was 12.5 (045.0), NIBUT was 10.4 (5.1-28.9) seconds, and Schirmer score was 8.0 (2.0-28.0) $\mathrm{mm}$. The prevalence of DED, preclinical dry eye, and predisposition to dry eye were $22.5 \%, 22.5 \%$, and $26.5 \%$, respectively, while the other $28.5 \%$ subjects were normal. The DED group had significantly lower Schirmer test results $(4.0$ [2-17] $\mathrm{mm})$ compared to all other groups. Older age ( $>70$ years) was associated with poorer NIBUT and Schirmer test results and a significant difference between age groups was observed, especially in males.

Conclusion: Dry eye is a common problem among the elderly population with cataracts in Indonesia. Almost half of the subjects presented with dry eye symptoms and either categorized as DED or preclinical dry eye, while more than one quarter were predisposed to dry eye. Care must be taken prior to cataract surgery to rule out existing dry eye with additional attention on those presenting without any symptoms as dry eye may develop after surgery.

Keywords: dry eye, elderly population, tear film, cataract, cross-sectional study

\section{Introduction}

Dry eye disease (DED) is one of most common ocular diseases and reason for primary eye care visits worldwide. Symptoms of dry eye may vary from mild ocular discomfort to severe fatigue, which may affect daily activities and quality of life. $^{1-3}$ The prevalence of dry eye ranged from $4.3 \%$ to $75 \%$ in several populationbased studies. $^{4-11}$ This large variation is attributed to the difference in study population and also the lack of standardized definition for the diagnosis of dry eye. ${ }^{2}$

There are limited reports of dry eye prevalence in Asia, especially those from Southeast Asia. Previous studies revealed that the prevalence of dry eye ranged from $21 \%$ to $73.5 \%$ using a symptoms questionnaire. ${ }^{4,6,8,11}$ There was a report of population-based data in a single province in a western area of Indonesia, with an 
average age of 37 years, who used self-diagnostic questionnaires without objective tests and the prevalence of dry eye was $27.5 \%{ }^{3}$ The information is even more limited on the prevalence of DED in the elderly population in Indonesia.

In daily clinical practice, tear film and dry eye assessment are often performed in symptomatic patients to confirm the diagnosis of dry eye. However, it must be kept in mind that dry eye symptoms and signs are not well associated, as reported in previous studies. ${ }^{12,13}$

As in Dry Eye Workshop II (DEWS), released in 2017, a subclassification of dry eye was recommended which described a wide range of possible dry eye subcategories. The classification scheme in DEWS II allowed for consideration of cases where patients exhibit dry eye symptoms without evidence of obvious signs, or present with marked signs without positive symptoms. ${ }^{14}$

DED is prevalent in older adults. Older adults are particularly susceptible to inadequate tear production because of lacrimal gland dysfunction, altered reflex secretion, diminished corneal sensation, or inflammatory destruction of lacrimal glands. ${ }^{15}$ Abnormalities in eyelid positioning, abnormal corneal sensation, and decreased blink reflex are significant contributors to rapid tear film break-up and evaporation and are seen increasingly in older adults. ${ }^{16,17}$ Inflammation and oxidative stress, which increase in aging, may also play a key role in dry eye development in the elderly. ${ }^{18}$ Notably, older men and women are almost twice as likely to have dry eyes compared with their younger counterparts. ${ }^{15}$ Large epidemiological studies noted that dry eye prevalence increases in women and men every five years after the age of 50, with greater prevalence in women compared to men. ${ }^{4,19}$

Cataracts are one of the most common causes of visual impairment in the world, and advancing age is the single most important risk factor for cataracts. ${ }^{20}$ Cataract surgery is the most common procedure performed by ophthalmic surgeons. Nevertheless, patients' dissatisfaction may arise during the postoperative period due to unresolved ocular surface disease, including dry eye. ${ }^{21}$ It is known that cataract surgery may induce or exacerbate a pre-existing dry eye condition. ${ }^{22}$ The incidence of dry eye after phacoemulsification has been reported to be $9.8 \%{ }^{23-25}$ The clinical importance of DED seems to be high considering the adverse effects on quality of life, however preoperative prevalence of dry eye in cataract patients has not been studied extensively.
With an increasingly aged population, increased life expectancy, and requirements of cataract surgery, the presence of DED must be anticipated and cannot be overlooked. Therefore, to obtain prevalence of dry eye and its subtypes in the elderly population planned to undergo cataract phacoemulsification surgery in Jakarta, Indonesia, we conducted this study.

\section{Patients and Methods}

This was a cross-sectional study of consecutive patients scheduled to undergo standard phacoemulsification cataract surgery at two JEC Eye Hospital sites in Jakarta, Indonesia, conducted between March to June 2017. Ethical approval for this study was waived by the Institutional Review Board (IRB) of JEC Eye Hospital because the research had very minimal risk of harm to subjects, if any. Specific examinations performed with intent for this study were not invasive. Based on these rationales, the IRB then dismissed the need for ethical approval. Nevertheless, informed consent was obtained from all individual participants and the study was in adherence to the tenets of the Declaration of Helsinki and all national laws.

Forty-nine patients of at least 50 years of age due for routine cataract phacoemulsification surgery were included in this study. Exclusion criteria were any intraocular surgery in the previous 3 months and laser vision correction in the previous year before patient enrollment. Additionally, patients with history of ocular allergy, coexisting pterygium, and patients who has been on routine use of artificial tears or lubricants were excluded.

Preoperatively, all eligible subjects underwent a series of tests which included a structured Ocular Surface Disease Index (OSDI) questionnaire, noninvasive tear film break up time (NIBUT), and Schirmer test without anesthesia. These objective tests were done on both eyes, however only the eye with the most severe NIBUT was used for analysis. The Schirmer test without anesthesia is a part of routine assessments for every patient due to undergo cataract surgery in our hospital. Whereas the OSDI questionnaire and NIBUT were performed purposely for this research.

\section{Data Collection}

Trained interviewers administered the OSDI questionnaire which consisted of 12 questions to assess any symptoms on the ocular surface along with other demographic data. The OSDI questionnaire has 3 assessment categories: ocular symptoms, visual function, and triggering or environmental 
factors. We modified the questionnaire by excluding question numbers 4 and 5 which assess the presence of impaired and poor vision, as shown in Figure 1, since it may be difficult to differentiate whether the cause of these symptoms was cataract alone or combined with dry eye conditions. A similar method was used in another study. ${ }^{18}$ The total OSDI score was calculated using the following formula: sum of all answered questions $\times 100 /$ total number of answered questions $\times 4$. The OSDI scores range from 0 to 100 . OSDI score $\geq 13$ indicates the presence of dry eye symptoms.

The NIBUT was evaluated using Tearscope ${ }^{\circledR}$ (Keeler Ltd, Windsor, UK) and was performed before the Schirmer test to avoid any unwanted interference. Time was measured using a built-in stopwatch in the device, and was started at the beginning of eyelid opening and stopped at the first appearance of grid distortion. The test was repeated three times and the average time was recorded. NIBUT was considered shortened if $<10$ seconds.

The Schirmer test was determined last without the aid of topical anesthesia. A precalibrated dry filter strip (Akriti Oculoplasty Logistics, Hyderabad, India) was placed at the junction of the middle and lateral thirds of the lower eyelid. The patient was asked to close his or her eyes. After 5 minutes, the strip was removed, and the amount of wetting was recorded. A Schirmer test result of $<10 \mathrm{~mm}$ was considered low.

Have you experienced any of the following during the last week:

\begin{tabular}{|l|c|c|c|c|c|}
\hline & $\begin{array}{c}\text { All of the } \\
\text { time }\end{array}$ & $\begin{array}{c}\text { Most of } \\
\text { the time }\end{array}$ & $\begin{array}{c}\text { Half of } \\
\text { the time }\end{array}$ & $\begin{array}{c}\text { Some of } \\
\text { the time }\end{array}$ & $\begin{array}{c}\text { None of } \\
\text { the time }\end{array}$ \\
\hline $\begin{array}{l}\text { 1. Eyes that are sensitive to } \\
\text { light? }\end{array}$ & 4 & 3 & 2 & 1 & 0 \\
\hline 2. Eyes that feel gritty & 4 & 3 & 2 & 1 & 0 \\
\hline 3. Painful or sore eyes? & 4 & 3 & 2 & 1 & 0 \\
\hline 4. Blurred vision? & \multicolumn{7}{|c|}{ NA } \\
\hline 5. Poor vision? & & & & & \\
\hline
\end{tabular}

Have problems with your eyes limited you in performing any of the following during the last week:

\begin{tabular}{|l|c|c|c|c|c|c|}
\hline & $\begin{array}{c}\text { All of the } \\
\text { time }\end{array}$ & $\begin{array}{c}\text { Most of } \\
\text { the time }\end{array}$ & $\begin{array}{c}\text { Half of } \\
\text { the time }\end{array}$ & $\begin{array}{c}\text { Some of } \\
\text { the time }\end{array}$ & $\begin{array}{c}\text { None of } \\
\text { the time }\end{array}$ & \\
\hline 6. Reading? & 4 & 3 & 2 & 1 & 0 & NA \\
\hline 7. Driving at night? & 4 & 3 & 2 & 1 & 0 & NA \\
\hline $\begin{array}{l}\text { 8. Working with a computer or } \\
\text { bank machine (ATM)? }\end{array}$ & 4 & 3 & 2 & 1 & 0 & NA \\
\hline 9. Watching TV? & 4 & 3 & 2 & 1 & 0 & NA \\
\hline
\end{tabular}

Have your eyes felt uncomfortable in any of the following situations during the last week:

\begin{tabular}{|l|c|c|c|c|c|c|}
\hline & $\begin{array}{c}\text { All of the } \\
\text { time }\end{array}$ & $\begin{array}{c}\text { Most of } \\
\text { the time }\end{array}$ & $\begin{array}{c}\text { Half of } \\
\text { the time }\end{array}$ & $\begin{array}{c}\text { Some of } \\
\text { the time }\end{array}$ & $\begin{array}{c}\text { None of } \\
\text { the time }\end{array}$ & \\
\hline 10. Windy conditions? & 4 & 3 & 2 & 1 & 0 & NA \\
\hline $\begin{array}{l}\text { 11. Places or areas with low } \\
\text { humidity (very dry)? }\end{array}$ & 4 & 3 & 2 & 1 & 0 & NA \\
\hline $\begin{array}{l}\text { 12. Areas that are air } \\
\text { conditioned? }\end{array}$ & 4 & 3 & 2 & 1 & 0 & NA \\
\hline
\end{tabular}

Figure I Ocular surface disease index (OSDI) questionnaire used for this study. Question 3 and 4 were not applicable (NA) for all subjects due to the possibility of interference from the cataract. 
Subjects were then categorized into one of 4 groups: DED, preclinical dry eye, predisposition to dry eye, and normal group. DED was defined if the patient had positive symptoms with shortened NIBUT, preclinical dry eye was defined as having symptoms with normal NIBUT, predisposition to dry eye was defined if the patient did not have symptoms but NIBUT was shortened, and the normal group had no symptoms with normal NIBUT.

\section{Statistical Analysis}

Statistical analysis was performed to describe the distribution of symptoms and signs and to explore the association between symptoms and clinical signs of dry eye. The normality of the data was checked with the Shapiro-Wilk test. The results were presented as mean $\pm \mathrm{SD}$ or median (range) according to the distribution of the data, and percentages. Quantitative data using independent-samples $t$-test or Mann-Whitney test and ANOVA or KruskalWallis test according to the data distribution. A $P$ value of $<0.05$ was considered statistically significant. All statistical analysis was done using IBM SPSS 23.0 (SPSS Inc., Chicago, IL).

\section{Results}

Forty-nine eyes from 49 subjects scheduled for cataract phacoemulsification surgery were analyzed. The age of the subjects ranged between 50 and 83 years and the ratio between male and female was 1:2. Twenty-two subjects $(44.8 \%)$ were categorized as symptomatic while the other 27 subjects $(55.2 \%)$ were asymptomatic. The prevalence of DED, preclinical dry eye, predisposition to dry eye and normal subjects were $22.5 \%, 22.5 \%, 26.5 \%$ and $28.5 \%$, respectively.

The demographic and clinical characteristics of each subtype are shown in Table 1. OSDI and NIBUT inevitably showed significant difference between all groups because categorization into each subtype was based on these parameters. However, in terms of OSDI, pairwise comparison showed that there was no significant difference between DED group and preclinical dry eye group neither between predisposition to the dry eye group and normal group. In terms of NIBUT, no significant difference was found between the DED group and predisposition to dry eye group or between the preclinical dry eye group and normal group. However, the Schirmer score was significantly lower in the DED group compared to the other dry eye subtypes.

To see if age affected the findings in our study, we performed additional analysis by dividing the subjects into two age groups, $\leq 70$ years and $>70$ years, as shown in Table 2. The percentage of subjects with DED was higher in the $>70$ years group. In the $\leq 70$ years group, most subjects (33.3\%) fell into the preclinical dry eye subtype. Subjects with older age $>70$ years also had significantly lower NIBUT and Schirmer scores.

Figure 2 illustrates the comparison of NIBUT between males and females in both age groups, whereas Figure 3 compares the Schirmer score. In NIBUT, the significant difference between the two age groups was observed only in male subjects $(\mathrm{p}=0.011)$ with NIBUT $16.0 \pm 6.6$ and $8.3 \pm 2.3$ seconds in the age group $\leq 70$ years old and $>70$ years old, respectively. We found similar results in terms of the Schirmer score. Significant difference was found only in male subjects $(\mathrm{p}=0.016)$ which were $10.0(4-28)$ and $4.0(2-10) \mathrm{mm}$, in age group $\leq 70$ years old and $>70$ years old, respectively.

\section{Discussion}

To the best of our knowledge, this is the first hospitalbased study to investigate the prevalence of DED in the

Table I Prevalence and Characteristics of Each Dry Eye Subtypes

\begin{tabular}{|c|c|c|c|c|c|}
\hline & \multicolumn{2}{|c|}{ Symptomatic $(n=22)$} & \multicolumn{2}{|l|}{ Asymptomatic $(n=27)$} & \multirow[t]{2}{*}{ P value } \\
\hline & DED $(n=I I)$ & Preclinical DE $(n=I I)$ & Predisposition to $D E(n=13)$ & Normal $(n=14)$ & \\
\hline Age (years) & $75.0(50-80)$ & $62.7 \pm 6.0$ & $66.8 \pm 5.6$ & $66.2 \pm 9.1$ & $0.173^{\mathrm{a}}$ \\
\hline \multicolumn{6}{|l|}{ Gender (n [\%]) } \\
\hline Male & $4(36.4)$ & $3(27.3)$ & $4(30.8)$ & $5(35.7)$ & \\
\hline Female & $7(63.6)$ & $8(72.7)$ & $9(69.2)$ & $9(64.3)$ & \\
\hline OSDI score & $30.5 \pm 7.9$ & $29.2(15.6-42.9)$ & $7.3 \pm 4.1$ & $5.4(0-12.5)$ & $<0.00 I^{\mathrm{a} *}$ \\
\hline NIBUT (seconds) & $7.9 \pm 1.5$ & $12.4(10.5-19.3)$ & $7.6 \pm 1.2$ & I5.I (10.4-28.9) & $<0.001^{\mathrm{a} *}$ \\
\hline Schirmer (mm) & $4.0(2-17)$ & $10.2 \pm 6.1$ & $11.1 \pm 7.0$ & $9.0(4-28)$ & $0.042^{\mathrm{a} *}$ \\
\hline
\end{tabular}

Notes: ${ }^{\text {a}}$ Kruskal-Wallis test; significance is indicated with asterisks.

Abbreviations: DE, dry eye; DED, dry eye disease; NIBUT, noninvasive tear break up time; OSDI, ocular surface disease index. 
Table 2 Prevalence and Characteristics of Each Dry Eye Subtypes According to Age Groups

\begin{tabular}{|l|l|l|l|}
\hline & $\begin{array}{l}\text { Age } \leq \mathbf{7 0} \\
\text { Years (n = 33) }\end{array}$ & $\begin{array}{l}\text { Age >70 } \\
\text { Years (n = 16) }\end{array}$ & $\begin{array}{l}\text { P } \\
\text { value }\end{array}$ \\
\hline $\begin{array}{l}\text { Gender (n [\%]) } \\
\text { Male }\end{array}$ & 9 & 7 & \\
Female & 24 & 9 & \\
\hline DE subtypes (n [\%]) & & & \\
DED & $4(12.1)$ & $7(43.8)$ & \\
Preclinical DE & $1 \mathrm{I}(33.3)$ & $0(0)$ & \\
Predisposition to DE & $8(24.3)$ & $5(31.2)$ & \\
Normal & $10(30.3)$ & $4(25.0)$ & \\
OSDI score & $12.5(0-42.9)$ & $10.4(0-45)$ & $0.669^{\mathrm{a}}$ \\
NIBUT (seconds) & $\mathrm{II} .6(6.2-28.9)$ & $8.8 \pm 3.1$ & $0.005^{\mathrm{a} *}$ \\
Schirmer (mm) & $10.0(2-28)$ & $5.0(2-20)$ & $0.03 \mathrm{I}^{\mathrm{a} *}$ \\
\hline
\end{tabular}

Notes: ${ }^{2}$ Mann-Whitney test; significance is indicated with asterisks. Abbreviations: DE, dry eye; DED, dry eye disease; NIBUT, noninvasive tear break up time; OSDI, ocular surface disease index.

elderly population in Indonesia. This is also the first study to evaluate the preoperative condition of dry eye and its subtypes in the Indonesian population. We strongly encourage further exploration as our findings may relate to the prevalence of post-cataract surgery dry eye.

Research into dry eye has been challenging as there are different diagnosis criteria, study population, the use of

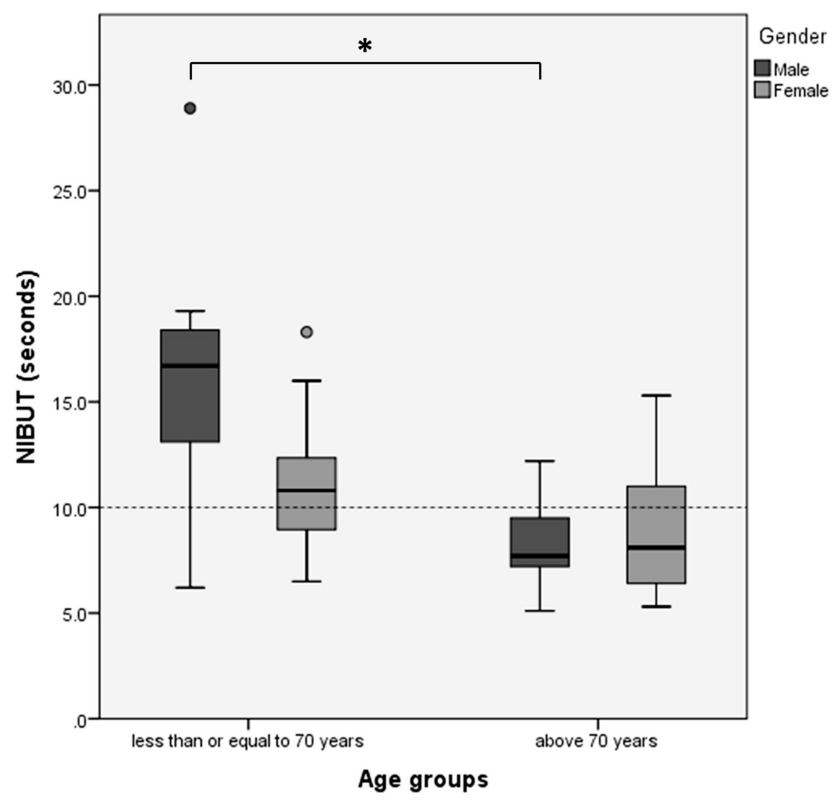

Figure 2 Difference of NIBUT in male and female subjects between age group $\leq 70$ years-old and $>70$-years-old. Significant difference of noninvasive break up time (NIBUT), marked with an asterisk $\left(^{*}\right)$, was observed only in male subjects between age group $\leq 70$-years-old and $>70$-years-old.

Notes: Statistical analysis with Student's T-test; significance is indicated with asterisks.

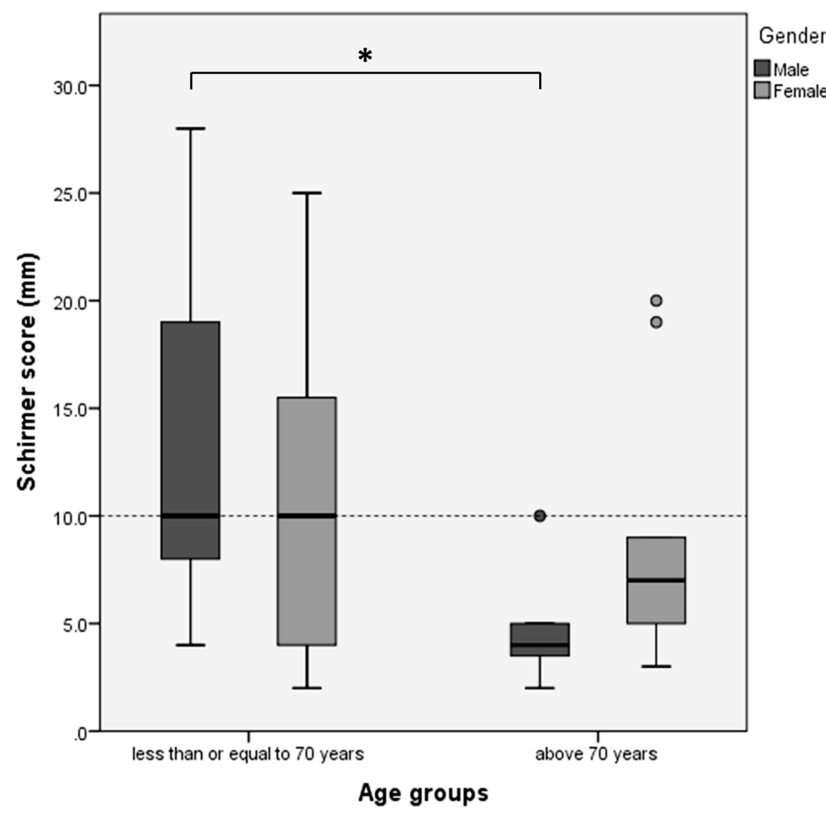

Figure 3 Difference of Schirmer score in male and female subjects between age group $\leq 70$-years-old and $>70$-years-old. Significant difference of Schirmer score was also found only in male subjects between age group $\leq 70$-years-old and $>70$-years-old. Notes: Statistical analysis with Student's T-test; significance is indicated with asterisks.

dry eye symptoms questionnaire, and objective tests. Although there have been many similar studies outside Indonesia, we felt the urge to perform dry eye evaluation because of the previously listed problems.

In alignment with the definition, the DED classification according to the DEWS II report recognizes the necessity of symptomatic involvement and the presence of associated ocular surface signs in making a diagnosis of DED. Besides DED, other subtypes were also described which consisted of symptoms without signs or preclinical dry eye and signs without symptoms or predisposition to dry eye. ${ }^{14}$

In our study we found that the prevalence of DED was $22.4 \%$. The same percentage of subjects fell into the preclinical dry eye subtype as they had symptoms but were not supported with decreased NIBUT. However, it needs to be kept in mind that there are other homeostasis markers for dry eye besides tear break up time. ${ }^{14}$ These markers are osmolarity and ocular surface staining, which unfortunately were not measured in this study. The prevalence of DED might be higher if subjects with preclinical dry eye were proven to have tear hyperosmolarity or ocular surface damage.

The clinical characteristics of dry eye subtypes indicated some interesting results. DED and preclinical dry eye subtypes both had moderate symptoms according to 
the OSDI. Despite having significantly lower NIBUT and Schirmer scores, DED subjects did not have more severe symptoms than those with preclinical dry eye. A recent systematic review reported that associations between DED signs and symptoms were low and inconsistent. ${ }^{26}$ Previous literatures have shown that signs of dry eye did not correlate well with either frequency or severity of symptoms.

Another interesting point was the explanation for positive symptoms in subjects with preclinical dry eye. Compared to DED subtypes, subjects with preclinical dry eye had significantly higher NIBUT and Schirmer scores, which were no different to those of normal subjects. The reason that may explain the presence of positive symptoms in this subtype is the possibility of abnormality in other homeostasis markers, such as tear hyperosmolarity or the presence of ocular surface damage. Tear hyperosmolarity was reported to be associated with DED symptoms and OSDI in previous studies. ${ }^{27,28}$ The presence of ocular surface staining may also contribute to increased symptoms, although studies showed conflicting significances. ${ }^{26}$ Another possibility is the presence of neuropathic pain if all homeostasis markers were proven negative. Discordance between symptoms and signs of dry eye with symptoms outweighing signs should prompt consideration of neuropathic mechanisms being involved. ${ }^{29,30}$

Subjects with predisposition to dry eye showed shortened NIBUT similar to DED group but without considerable symptoms. Compared to DED subtype, these subjects had higher Schirmer scores. As previously mentioned, dry eye symptoms and signs might not be well-correlated. However, previous studies show that a low Schirmer result was significantly associated with dry-eye symptoms in this elderly Asian population, ${ }^{31}$ which is similar to our study. It is also important to note that some dry eye conditions were associated with decreased corneal sensitivity which might explain the absence of symptoms. Baseline subbasal nerve fiber length was significantly lower in patients with dry eye compared to normal subjects, which is associated with decreased corneal sensitivity. ${ }^{32}$ This might explain why a group of patients manifests signs of dry eye yet remains asymptomatic.

The association between age and dry eye was also observed in this study. There was a likelihood of shifting from preclinical dry eye towards DED and from normal towards predisposition to dry eye in older age. Tear film stability was poorer and aqueous production was lower in older age. Previous studies mentioned that decreased tear production is a consequence of lacrimal gland dysfunction, altered reflex secretion, diminished corneal sensation, or lacrimal gland destruction due to inflammation in aging population. ${ }^{15}$ A similar result was seen in the study by Ozdemir et $\mathrm{al}^{33}$ in which NIBUT was significantly shorter in older age. One factor that may also affect this condition in the elderly population is the tear evaporation rate. Eyelid abnormalities such as laxity or lagophthalmos, meibomian gland dysfunction, and decreased blinking reflex were considered significant contributors to NIBUT shortening and most commonly seen in the elderly. ${ }^{16,17,34}$

These changes were markedly seen in males. This is in contrary with the results of most studies. Ozdemir et al found that age-related reduction of TBUT and Schirmer score was parallel in both genders. ${ }^{33}$ Generally, male had higher prevalence of MGD and older male above 70-yearsold had higher incidence of meibomian gland dropout, which might explain the significantly shorter TBUT in older groups. ${ }^{35}$

Data showed that women have a higher DED prevalence compared to men. Large-scale epidemiological studies carried out in the United States have shown that the rate of DED in women over 50-years-old is nearly double that in men over 50 , at $7 \%$ and $4 \%$, respectively. ${ }^{6}$ The high prevalence of DED among women is also related to hormonal changes as postmenopausal women are proven to have higher incidence of DED. ${ }^{36}$ The average menopausal age in the Indonesian female population was 50- to 51-yearsold. ${ }^{37}$ Compared to women, DED begins in men at later age. The prevalence of DED in men increased with age, from $3.9 \%$ among men 50- to 54-years-old to $7.67 \%$ among men 80 years and older. ${ }^{8}$ Aging in men is accompanied by a progressive, but individually variable decline of androgen levels. Healthy men over 60-years-old may present with serum levels below the range for young men. ${ }^{38}$ Androgen is important in the development and function of the lacrimal glands, meibomian glands, cornea and conjunctiva. A shortage can cause lacrimal gland dysfunction and aqueous tear deficiency, development of MGD, and increased conjunctival and corneal staining. ${ }^{35}$ This might explain the findings of significantly lower NIBUT and Schirmer test results in male subjects over 70-years-old, compared to the younger group in our study.

This study is certainly not without limitations. The first and the major drawback is that other parameters such as ocular surface staining, tear osmolarity, and meibomian gland dysfunction, which could have provided valuable information, were not assessed, this is followed by the small number of subjects. Second, other potential biases, 
such as the use of topical or systemic medications and presence of systemic disease which might interfere with the tear film, were not assessed in this study. Third, the imbalance between male and female subjects might also affect the result.

\section{Conclusion}

Our study has shown that dry eye is a common problem among the elderly population with cataracts in Indonesia. We found that the prevalence of DED and preclinical dry eye was $22.45 \%$ in each subtype. However, $26.53 \%$ subjects were predisposed to dry eye. Care must be taken in all dry eye patients, with additional attention to those presenting without any symptoms, as dry eye may develop after surgery. Further studies with prospective design, larger sample size, and complete tear film and ocular surface evaluation is warranted to provide evident results of DED prevalence and its effect on the outcomes of cataract surgery in the Indonesian population.

\section{Disclosure}

The authors report no conflicts of interest in this work.

\section{References}

1. Moss SE, Klein R, Klein BE. Long-term incidence of dry eye in an older population. Optom Vis Sci. 2008;85(8):668-674.

2. Tong L, Waduthantri S, Wong TY, et al. Impact of symptomatic dry eye on vision related daily activities: the Singapore Malay Eye Study Eye (Lond). 2010;24(9):1486-1491. doi:10.1038/eye.2010.67

3. Lee AJ, Lee J, Saw SM, et al. Prevalence and risk factors associated with dry eye symptoms: a population based study in Indonesia. $\mathrm{Br} \mathrm{J}$ Ophthalmol. 2002;86(12):1347-1351. doi:10.1136/bjo.86.12.1347

4. Chia EM, Mitchell P, Rochtchina E, Lee AJ, Maroun R, Wang JJ. Prevalence and associations of dry eye syndrome in an older population: the Blue Mountains Eye Study. Clin Experiment Ophthalmol. 2003;31(3):229-232. doi:10.1046/j.1442-9071.2003.00634.x

5. Lin PY, Tsai SY, Cheng CY, Liu JH, Chou P, Hsu WM. Prevalence of dry eye among an elderly Chinese population in Taiwan: the Shihpai Eye Study. Ophthalmology. 2003;110(6):1096-1101. doi:10.1016/ S0161-6420(03)00262-8

6. Schaumberg DA, Sullivan DA, Buring JE, Dana MR. Prevalence of dry eye syndrome among US women. Am J Ophthalmol. 2003;136 (2):318-326. doi:10.1016/S0002-9394(03)00218-6

7. Uchino M, Schaumberg DA, Dogru M, et al. Prevalence of dry eye disease among Japanese visual display terminal users. Ophthalmology. 2008;115(11):1982-1988. doi:10.1016/j.ophtha.2008.06.022

8. Schaumberg DA, Dana R, Buring JE, Sullivan DA. Prevalence of dry eye disease among US men: estimates from the physicians' health studies. Arch Ophthalmol. 2009;127(6):763-768. doi:10.1001/ archophthalmol.2009.103

9. Viso E, Rodriguez-Ares MT, Gude F. Prevalence of and associated factors for dry eye in a Spanish adult population (the Salnes Eye Study). Ophthalmic Epidemiol. 2009;16(1):15-21. doi:10.1080/ 09286580802228509

10. Uchino M, Dogru M, Yagi Y, et al. The features of dry eye disease in a Japanese elderly population. Optom Vis Sci. 2006;83(11):797-802. doi:10.1097/01.opx.0000232814.39651.fa
11. Stapleton F, Alves M, Bunya VY, et al. TFOS DEWS II epidemiology report. Ocul Surf. 2017;15(3):334-365. doi:10.1016/j.jtos.2017.05.003

12. Schein OD, Tielsch JM, Munõz B, Bandeen-Roche K, West S. Relation between signs and symptoms of dry eye in the elderly: a population-based perspective. Ophthalmology. 1997;104(9):13951401. doi:10.1016/S0161-6420(97)30125-0

13. McCarty CA, Bansal AK, Livingston PM, Stanislavsky YL, Taylor HR. The epidemiology of dry eye in Melbourne, Australia. Ophthalmology. 1998;105(6):1114-1119. doi:10.1016/S0161-6420 (98) $96016-X$

14. Craig JP, Nichols KK, Akpek EK, et al. TFOS DEWS II definition and classification report. Ocul Surf. 2017;15(3):276-283. doi:10. 1016/j.jtos.2017.05.008

15. Sharma A, Hindman HB. Aging: a predisposition to dry eyes. $J$ Ophthalmol. 2014;2014:781683. doi:10.1155/2014/781683

16. Damasceno RW, Osaki MH, Dantas PE, Belfort R. Involutional entropion and ectropion of the lower eyelid: prevalence and associated risk factors in the elderly population. Ophthalmic Plast Reconstr Surg. 2011;27(5):317-320. doi:10.1097/IOP.0b013e3182115229

17. Murphy PJ, Patel S, Kong N, Ryder REJ, Marshall J. Noninvasive assessment of corneal sensitivity in young and elderly diabetic and nondiabetic subjects. Invest Ophthalmol Vis Sci. 2004;45(6):17371742. doi:10.1167/iovs.03-0689

18. Dogru M, Wakamatsu T, Kojima T, et al. The role of oxidative stress and inflammation in dry eye disease. Cornea. 2009;28(1):S70-S74. doi:10.1097/ICO.0b013e3181ae8689

19. Moss SE, Klein R, Klein BE. Incidence of dry eye in an older population. Arch Ophthalmol. 2004;122:369-373. doi:10.1001/ archopht.122.3.369

20. Singh S, Pardhan S, Kulothungan V, et al. The prevalence and risk factors for cataract in rural and urban India. Indian J Ophthalmol. 2019;67(4):477-483. doi:10.4103/ijo.IJO_1127_17

21. Cho YK, Kim MS. Dry eye after cataract surgery and associated intraoperative risk factors. Korean J Ophthalmol. 2009;23(2):65-73. doi:10.3341/kjo.2009.23.2.65

22. Oh T, Jung Y, Chang D, Kim J, Kim H. Changes in the tear film and ocular surface after cataract surgery. Jpn J Ophthalmol. 2012;56 (2):113-118. doi:10.1007/s10384-012-0117-8

23. Yao K, Bao Y, Ye J, et al. Efficacy of $1 \%$ carboxymethylcellulose sodium for treating dry eye after phacoemulsification: results from a multicenter, open-label, randomized, controlled study. $B M C$ Ophthalmol. 2015;15:28. doi:10.1186/s12886-015-0005-3

24. Han KE, Yoon SC, Ahn JM, et al. Evaluation of dry eye and meibomian gland dysfunction after cataract surgery. Am J Ophthalmol. 2014;157(6):1144-1150.e1. doi:10.1016/j.ajo.2014.02.036

25. Kasetsuwan N, Satitpitakul V, Changul T, Jariyakosol S, Wedrich A. Incidence and pattern of dry eye after cataract surgery. PLoS One. 2013;8(11):e78657. doi:10.1371/journal.pone.0078657

26. Bartlett JD, Keith MS, Sudharshan L, Snedecor SJ. Associations between signs and symptoms of dry eye disease: a systematic review. Clin Ophthalmol. 2015;9:1719-1730.

27. Yi HC, Lee YP, Shin YJ. Influence of nasal tear osmolarity on ocular symptoms related to dry eye disease. $A m$ J Ophthalmol. 2018;189:71-76. doi:10.1016/j.ajo.2018.02.008

28. Kim M, Kim HS, Na KS. Correlation between tear osmolarity and other ocular surface parameters in primary sjögren's syndrome. Korean $J$ Ophthalmol. 2017;31(1):25-31. doi:10.3341/kjo.2017.31.1.25

29. Mcmonnies CW. The potential role of neuropathic mechanisms in dry eye syndromes. J Optom. 2017;10(1):5-13. doi:10.1016/j.optom. 2016.06.002

30. Galor A, Moein HR, Lee C, et al. Neuropathic pain and dry eye. Ocul Surf. 2018;16(1):31-44. doi:10.1016/j.jtos.2017.10.001

31. Lin PY, Cheng CY, Hsu WM, et al. Association between symptoms and signs of dry eye among an elderly Chinese population in Taiwan: the Shihpai Eye Study. Invest Ophthalmol Vis Sci. 2005;46(5):15931598. doi:10.1167/iovs.04-0864 
32. Kheirkhah A, Dohlman TH, Amparo F, et al. Effects of corneal nerve density on the response to treatment in dry eye disease. Ophthalmology. 2015;122(4):662-668. doi:10.1016/j.ophtha.2014.11.006

33. Ozdemir M, Temizdemir H. Age- and gender-related tear function changes in normal population. Eye (Lond). 2010;24(1):79-83. doi:10.1038/eye.2009.21

34. Villani E, Canton V, Magnani F, Viola F, Nucci P, Ratiglia R. The aging meibomian gland: an in vivo confocal study. Invest Ophthalmol Vis Sci. 2013;54(7):4735-4740. doi:10.1167/iovs.13-11914

35. Sullivan D, Rocha E, Aragona P, et al. TFOS DEWS II sex, gender, and hormones report. Ocul Surf. 2017;15(3):284-333. doi:10.1016/j. jtos.2017.04.001
36. Peck T, Olsakovsky L, Aggarwal S. Dry eye syndrome in menopause and perimenopausal age group. J Midlife Health. 2017;8(2):51-54. doi:10.4103/jmh.JMH_41_17

37. Samil RS, Wishnuwardhani SD. Health of Indonesian women citydwellers of perimenopausal age. Maturitas. 1994;19(3):191-197. doi:10.1016/0378-5122(94)90071-X

38. Kaufman JM, Vermeulen A. The decline of androgen levels in elderly men and its clinical and therapeutic implications. Endocr Rev. 2005;26(6):833-876.
Clinical Ophthalmology

\section{Publish your work in this journal}

Clinical Ophthalmology is an international, peer-reviewed journal covering all subspecialties within ophthalmology. Key topics include: Optometry; Visual science; Pharmacology and drug therapy in eye diseases; Basic Sciences; Primary and Secondary eye care; Patient Safety and Quality of Care Improvements. This journal is indexed on PubMed

Submit your manuscript here: https://www.dovepress.com/clinical-ophthalmology-journal

\section{Dovepress}

Central and CAS, and is the official journal of The Society of Clinical Ophthalmology (SCO). The manuscript management system is completely online and includes a very quick and fair peer-review system, which is all easy to use. Visit http://www.dovepress.com/ testimonials.php to read real quotes from published authors. 